\section{Comparative analysis of SGLT-2 expression in renal vasculitis and lupus nephritis}

The nephroprotective efficacy of sodium-glucose cotransporter-2 (SGLT-2) inhibitors (SGLT-2i) in patients with chronic kidney disease, with or without type 2 diabetes, has already been described. ${ }^{1}$ Although patients with autoimmune diseases were excluded from large renal outcome trials, the use of SGLT-2i in antineutrophil cytoplasmic antibody-associated vasculitis (AAV) and systemic lupus erythematodes (SLE) for nephroprotection has increasingly been emphasised. ${ }^{2-4}$ While SGLT-2 was studied in diabetic nephropathy and IgA nephropathy (IgAN), the presence of SGLT-2 specifically in renal vasculitis and lupus nephritis has not been described yet. ${ }^{5}$

In renal vasculitis and lupus nephritis, SGLT-2 was detectable and predominantly localised to the tubulointerstitial as compared with the glomerular compartment (figure 1A). For comparative analysis, we next extracted transcriptome data sets for SGLT-2 expression (encoded by SLC5A2), specifically from microdissected tubulointerstitial (31 healthy controls, 17 with diabetic nephropathy, 25 with IgAN, 21 with renal vasculitis and 32 with lupus nephritis) and glomerular compartments (21 healthy controls, 12 with diabetic nephropathy, 27 with IgAN, 23 with renal vasculitis and 32 with lupus nephritis, www.nephroseq.org, January 2022, University of Michigan, Ann Arbor, Michigan, online supplemental tables 1 and 2). ${ }^{6}$ In renal vasculitis and lupus nephritis, tubulointerstitial SLC5A2 mRNA expression was significantly higher as compared with the glomerular compartment $(\mathrm{p}<0.0001$, figure $1 \mathrm{~B})$, with no sex differences for tubulointerstitial $(\mathrm{p}=0.7)$ or glomerular SLC5A2 expression $(\mathrm{p}=0.4$, figure $1 \mathrm{C})$. Interestingly, kidney function positively correlated with particularly tubulointerstitial SLC5A2 mRNA expression in renal vasculitis and lupus nephritis (figure 1D and online supplemental figure 1A,B). Compared with healthy control kidneys, a significant reduction of tubulointerstitial SLC5A2 mRNA expression levels was detectable in diabetic nephropathy $(p=0.01)$, while IgAN, renal vasculitis and lupus nephritis did not significantly differ $(p=0.6$ for all comparisons, figure $1 \mathrm{E}$ and online supplemental figure 2A). In contrast, glomerular SLC5A2 was significantly reduced in diabetic nephropathy $(p=0.0005)$, IgAN $(p=0.0003)$, renal vasculitis and lupus nephritis $(\mathrm{p}<0.0001$ for both comparisons, figure 1E). Interestingly, no association between kidney function and SLC5A2 was observed in diabetic nephropathy and IgAN (online supplemental figure $3 \mathrm{~A}, \mathrm{~B})$. Gene set enrichment analysis in the whole data set (including 201 tubulointerstitial and 199 glomerular compartments) linking SLC5A2 expression to potential signalling pathways revealed positive associations with lipid metabolism by peroxisome proliferator-activated receptor (PPAR)- $\alpha$ and the tricarboxylic acid (TCA) cycle (figure $1 \mathrm{~F}$ and online supplemental table 3). Glomerular SLC5A2 mRNA expression correlated with protein and fatty acid metabolism (figure $1 \mathrm{~F}$ and online supplemental table 4).

In summary, this comparative analysis revealed predominant tubulointerstitial expression of SGLT-2 in renal vasculitis and lupus nephritis. Moreover, worse kidney function correlated with loss of tubulointerstitial SGLT-2 expression particularly in renal vasculitis and lupus nephritis.
Therefore, nephroprotection by SGLT-2i could be effective especially once the initial phase of remission induction in renal vasculitis and lupus nephritis is achieved and kidney function stabilised. Finally, this is the first report linking SGLT-2 to distinct metabolic signalling pathways in the kidney that could also be relevant for autoimmune diseases including AAV and SLE. In general, management of renal complications in AAV and SLE involves immunosuppressive and anti-inflammatory treatment, and also an approach towards nephroprotection and cardiovascular risk reduction. ${ }^{2-4}$ SGLT-2i exerts unequivocal nephroprotective effects by reducing glomerular hyperfiltration and albuminuria, tubular injury, loss of kidney function and incidence of acute kidney injury. These effects suggest that SGLT-2i is an ideal therapeutic approach also for patients with AAV and SLE, especially when renal and cardiac complications already manifested. Because of limited information about treatment at time of kidney biopsy or detailed histopathology in the current study, we emphasise future studies regarding SGLT-2, particularly in autoimmune diseases with renal involvement (including AAV and SLE).

Samy Hakroush, ${ }^{1}$ Desiree Tampe, ${ }^{2}$ Ingmar Alexander Kluge, ${ }^{1}$ Eva Baier, ${ }^{2}$ Peter Korsten $\odot^{2}$ ' Björn Tampe $\circledast^{2}$

${ }^{1}$ Institute of Pathology, University Medical Center Göttingen, Göttingen, Germany ${ }^{2}$ Department of Nephrology and Rheumatology, University Medical Center Göttingen, Göttingen, Germany

Correspondence to Dr Björn Tampe, Department of Nephrology and Rheumatology, University Medical Center Göttingen, Göttingen 37075, Germany; bjoern.tampe@med.uni-goettingen.de

\section{Handling editor Josef S Smolen}

Twitter Peter Korsten @pekor002

Contributors BT conceived the letter, analysed data and wrote the manuscript. SH and IAK performed histological analysis, PK provided samples of lupus nephritis. DT and EB performed gene set enrichment analysis. All authors reviewed and approved the manuscript's content before submission.

Funding This research was funded by the Research program, University Medical Center, University of Göttingen, grant number 1403720. We also acknowledge support by the Open Access Publication Funds of the Göttingen University.

Competing interests None declared.

Patient consent for publication Not applicable.

Ethics approval This study involves human participants and was conducted according to the guidelines of the Declaration of Helsinki, and approved by the Institutional Review Board of the University Medical Center Göttingen, Germany (28/9/17). Additional data were extracted from Nephroseq Research Edition (Ann Arbor, MI: University of Michigan; available from: https://www.nephroseq.org/). Participants gave informed consent to participate in the study before taking part.

Provenance and peer review Not commissioned; externally peer reviewed.

Supplemental material This content has been supplied by the author(s). It has not been vetted by BMJ Publishing Group Limited (BMJ) and may not have been peer-reviewed. Any opinions or recommendations discussed are solely those of the author(s) and are not endorsed by BMJ. BMJ disclaims all liability and responsibility arising from any reliance placed on the content. Where the content includes any translated material, BMJ does not warrant the accuracy and reliability of the translations (including but not limited to local regulations, clinical guidelines, terminology, drug names and drug dosages), and is not responsible for any error and/or omissions arising from translation and adaptation or otherwise.

\section{(2) OPEN ACCESS}

Open access This is an open access article distributed in accordance with the Creative Commons Attribution Non Commercial (CC BY-NC 4.0) license, which permits others to distribute, remix, adapt, build upon this work non-commercially, 
A

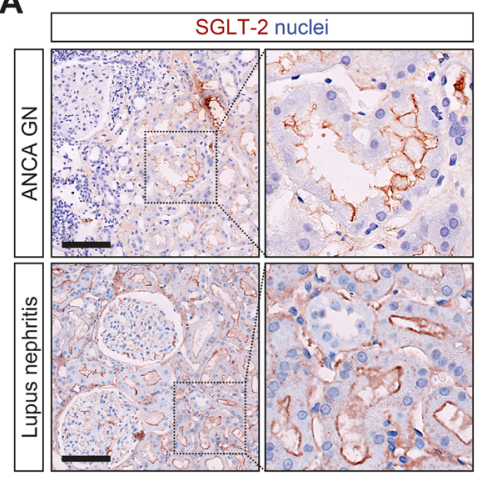

B

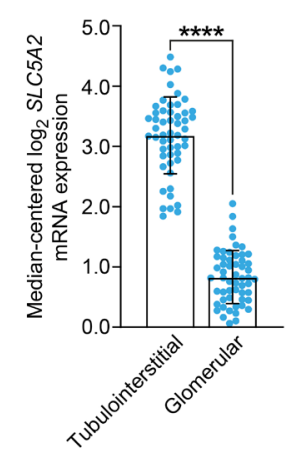

C

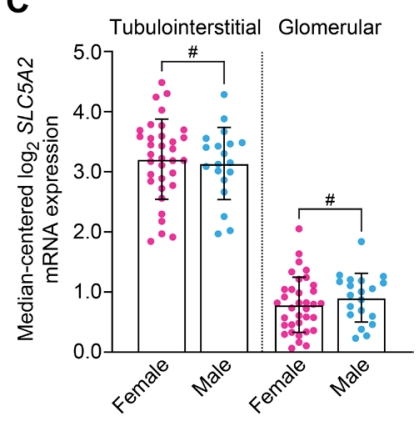

D

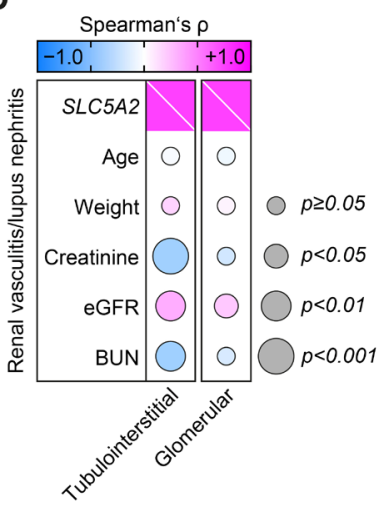

E

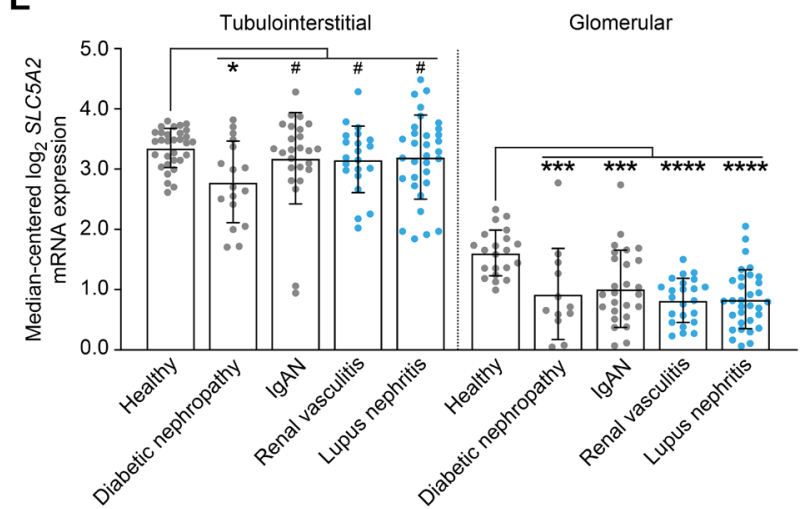

F
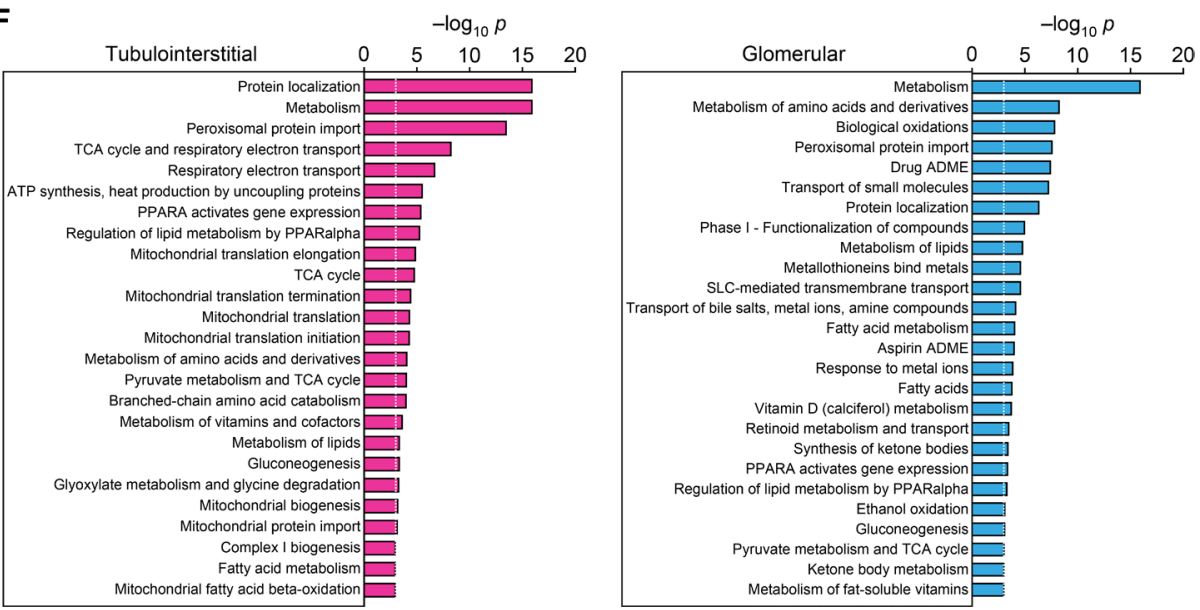

Figure 1 Comparative analysis of SGLT-2 expression in renal vasculitis and lupus nephritis. (A) Immunostaining for SGLT-2 in renal vasculitis (ANCA GN) and lupus nephritis, counterstaining was performed by using hematoxylin (scale bars $100 \mu \mathrm{m}$ ). SGLT-2 was detectable and predominantly localised to the luminal brush border of the proximal tubule of the renal cortex in renal vasculitis and lupus nephritis. (B) Direct comparison between tubulointerstitial and glomerular SLC5A2 mRNA expression in renal vasculitis (tubulointerstitial: $n=21$, glomerular: $n=23$ ) and lupus nephritis (tubulointerstitial: $n=32$, glomerular: $n=32$ ). In renal vasculitis and lupus nephritis, tubulointerstitial $S L C 5 A 2$ mRNA expression was significantly higher as compared with the glomerular compartment. Median centred $\log _{2}$ mRNA expression levels are shown, comparison of groups was performed using unpaired $t$ test $\left.{ }^{* * * *} p<0.0001\right)$. (C) No significant sex differences in SLC5A2 mRNA expression in microdissected tubulointerstitial (female: $n=34$, male: $n=19$ ) and glomerular compartments (female: $n=35$, male: $n=20$ ) of renal vasculitis and lupus nephritis. Median centred $\log _{2}$ mRNA expression levels are shown, comparison of groups was performed using unpaired t test ("not significant). (D) Correlations between tubulointerstitial and glomerular SLC5A2 mRNA expression levels, clinical and laboratory markers of kidney function in renal vasculitis and lupus nephritis are shown by heatmap reflecting mean values of Spearman's $\rho$, circle size represents significance level. (E) Median centred $\log _{2}$ mRNA expression levels of SLC5A2 in microdissected tubulointerstitial (healthy controls: $n=31$, diabetic nephropathy: $n=17, \lg A N: n=25$, renal vasculitis: $n=21$, lupus nephritis: $n=32$ ) and glomerular compartments (healthy controls: $n=21$, diabetic nephropathy: $n=12$, IgAN: $n=27$, renal vasculitis: $n=23$, lupus nephritis: $n=32$ ) are shown. Comparisons of groups were performed using one-way analysis of variance (ANOVA) and correction for multiple comparisons by Holm-Šíák test $\left({ }^{*} p<0.05,{ }^{* *} p<0.01,{ }^{* * *} p<0.001,{ }^{*}\right.$ not significant). (F) Entities $-\log _{10} p$ values of signalling pathways separated for gene enrichment associated with either tubulointerstitial or glomerular $S L C 5 A 2 \mathrm{mRNA}$ expression are shown (the dotted lines correspond to the predefined threshold value of $\mathrm{p} \leq 0.001)$. Tubulointerstitial $S L C 5 A 2$ mRNA expression levels are associated with enrichment of genes predominantly involved in lipid metabolism by peroxisome proliferator-activated receptor (PPAR)- $\alpha$ and the tricarboxylic acid (TCA) cycle, glomerular SLC5A2 correlated with protein and fatty acid metabolism . 
and license their derivative works on different terms, provided the original work is properly cited, appropriate credit is given, any changes made indicated, and the use is non-commercial. See: http://creativecommons.org/licenses/by-nc/4.0/.

(C) Author(s) (or their employer(s)) 2022. Re-use permitted under CC BY-NC. No commercial re-use. See rights and permissions. Published by BMJ.

- Additional supplemental material is published online only. To view, please visit the journal online (http://dx.doi.org/10.1136/annrheumdis-2022-222167).

\section{A) Check for updates}

To cite Hakroush S, Tampe D, Kluge IA, et al. Ann Rheum Dis Epub ahead of print: [please include Day Month Year]. doi:10.1136/annrheumdis-2022-222167

Received 15 January 2022

Accepted 19 February 2022

Ann Rheum Dis 2022;0:1-3. doi:10.1136/annrheumdis-2022-222167

ORCID iDs

Peter Korsten http://orcid.org/0000-0001-6065-5680

Björn Tampe http://orcid.org/0000-0002-4357-9863

\section{REFERENCES}

1 Heerspink HJL, Stefánsson BV, Correa-Rotter R, et al. Dapagliflozin in patients with chronic kidney disease. N Engl J Med 2020;383:1436-46.

2 Säemann M, Kronbichler A. Call for action in ANCA-associated vasculitis and lupus nephritis: promises and challenges of SGLT-2 inhibitors. Ann Rheum Dis 2021. doi:10.1136/annrheumdis-2021-221474. [Epub ahead of print: 29 Nov 2021].

3 Patoulias D. Correspondence on 'Call for action in ANCA-associated vasculitis and lupus nephritis: promises and challenges of SGLT-2 inhibitors' by Säemann and Kronbichler. Ann Rheum Dis 2022. doi:10.1136/annrheumdis-2021-221953. [Epub ahead of print: 13 Jan 2022].

4 Säemann M, Kronbichler A. Response to: Correspondence on 'Call for action in ANCA-associated vasculitis and lupus nephritis: promises and challenges of SGLT-2 inhibitors' by Säemann and Kronbichler. Ann Rheum Dis 2022. doi:10.1136/ annrheumdis-2021-221982. [Epub ahead of print: 13 Jan 2022].

5 Srinivasan Sridhar V, Ambinathan JPN, Kretzler M, et al. Renal SGLT mRNA expression in human health and disease: a study in two cohorts. Am I Physiol Renal Physiol 2019:317:F1224-30.

$6 \mathrm{Ju}$ W, Nair V, Smith S, et al. Tissue transcriptome-driven identification of epidermal growth factor as a chronic kidney disease biomarker. Sci Trans/ Med 2015;7:316ra193. 